\title{
Gravin regulates mesodermal cell behavior changes required for axis elongation during zebrafish gastrulation
}

\author{
Douglas C. Weiser, Ujwal J. Pyati, ${ }^{1}$ and David Kimelman ${ }^{2}$ \\ Department of Biochemistry, University of Washington, Seattle, Washington 98195, USA
}

\begin{abstract}
Convergent extension of the mesoderm is the major driving force of vertebrate gastrulation. During this process, mesodermal cells move toward the future dorsal side of the embryo, then radically change behavior as they initiate extension of the body axis. How cells make this transition in behavior is unknown. We have identified the scaffolding protein and tumor suppressor Gravin as a key regulator of this process in zebrafish embryos. We show that Gravin is required for the conversion of mesodermal cells from a highly migratory behavior to the medio-laterally intercalative behavior required for body axis extension. In the absence of Gravin, paraxial mesodermal cells fail to shut down the protrusive activity mediated by the Rho/ROCK/ Myosin II pathway, resulting in embryos with severe extension defects. We propose that Gravin functions as an essential scaffold for regulatory proteins that suppress the migratory behavior of the mesoderm during gastrulation, and suggest that this function also explains how Gravin inhibits invasive behaviors in metastatic cells.
\end{abstract}

[Keywords: AKAP; convergence and extension; gastrulation; Gravin; Rho; zebrafish]

Supplemental material is available at http://www.genesdev.org.

Received January 24, 2007; revised version accepted May 2, 2007.

Vertebrate gastrulation involves a tightly regulated series of cellular rearrangements that coordinate the movements of the three germ layers (endoderm, mesoderm, and ectoderm) to establish the final embryonic body plan (Keller 2002). This process requires a very precise regulation of cell polarity, cell migration, and cell division. One of the major driving forces of vertebrate gastrulation is convergent extension, a mechanism in which mesodermal cells move toward the future dorsal side of the embryo and then intercalate between neighboring cells, resulting in an overall dorsoventral narrowing (convergence) and anteroposterior lengthening (extension) of the embryo (Heisenberg and Tada 2002; Keller 2002; SolnicaKrezel 2006).

Mesodermal cells in the zebrafish embryo involved in convergent extension undergo a series of changes starting with a slow dorsal-directed migration, a faster migration as they get closer to the future dorsal side, and then intercalation as they approach the dorsal midline /Concha and Adams 1998; Jessen et al. 2002; Sepich et al. 2005; Solnica-Krezel 2006). As the mesodermal cells change their behaviors, they undergo a series of morpho-

${ }^{1}$ Present address: Department of Pediatric Oncology, Dana-Farber Cancer Institute, Boston, MA 02115, USA.

${ }^{2}$ Corresponding author.

E-MAIL kimelman@u.washington.edu; FAX (206) 616-8676.

Article is online at http://www.genesdev.org/cgi/doi/10.1101/gad.1535007. logical changes in cell shape and protrusive activity (Concha and Adams 1998). During early gastrulation, mesodermal cells have an overall rounded phenotype and extend transient lamellipodia and bleb-like protrusions with random orientation /Concha and Adams 1998; Sepich et al. 2005). By mid- to late gastrulation, cells become more polarized with lamellipodia that become stabilized and mediolaterally oriented. These cells then migrate in a highly dorsally biased manner. Later in gastrulation, mesodermal cells become tightly packed on the dorsal side of the embryo. These cells, through contact inhibition of migratory behavior, become elongated and firmly attached to their neighboring cells, reduce protrusions, and begin to medio-laterally intercalate (Concha and Adams 1998). How mesodermal cells make transitions between these different behaviors is largely unknown (Solnica-Krezel 2006).

Much of our understanding of the molecular control of gastrulation comes from the discovery that convergent extension is regulated by the noncanonical Wnt pathway (Veeman et al. 2003). The noncanonical Wnt pathway is the vertebrate version of the planar cell polarity (PCP) pathway that controls epithelial polarity in Drosophila (Mlodzik 2002). The vertebrate PCP pathway is activated by the noncanonical Wnts, Wnt5a (Kilian et al. 2003), Wnt4 (Matsui et al. 2005), and Wnt11 (Heisenberg et al. 2000). Importantly, either gain or loss of function of positive (Wallingford et al. 2000; Topczewski et al. 2001; 
Jessen et al. 2002) or negative regulators (Carreira-Barbosa et al. 2003) of PCP signaling results in severe gastrulation defects, indicating that precise control of cell behavior is required for convergence and extension to function properly. The noncanonical Wnts bind to the Frizzled receptor and promote membrane localization of Dishevelled (Wallingford et al. 2000), which then activates several downstream PCP effectors, including the small GTPases Rho (Habas et al. 2001), Rac (Habas et al. 2003), and Cdc42 (Choi and Han 2002; Matsui et al. 2005). This Rho family of G-proteins mediates many of the effects of the PCP pathway during gastrulation (Marlow et al. 2002; Habas et al. 2003) by affecting cell polarity, cell shape changes, and movement (Hall 1998). While most work on small G-proteins during gastrulation has focused on the role of noncanonical Wnts in controlling their activity (Habas et al. 2003), several other regulators of Rho or Rac are required for proper gastrulation. These include Has2-dependent (Bakkers et al. 2004), Fyn/Yes-dependent (Jopling and den Hertog 2005), and G $\alpha 12 / \mathrm{G} \alpha 13$-dependent pathways (Lin et al. 2005) as well as several guanine nucleotide exchange factors, which can directly activate small G-proteins (Daggett et al. 2004; Miyakoshi et al. 2004). With several distinct signaling pathways converging on the Rho family of GTPases as well as other intracellular factors involved in morphogenesis, precise integration of these pathways is essential for proper cell behavior during gastrulation.

One major mechanism of controlling multiple signaling pathways inside the cell is through scaffolding factors that can coordinate combinations of different intracellular regulatory proteins. Gravin, also called AKAP12, AKAP250, and SSeCKS, was originally identified as a tumor suppressor down-regulated in Src- or Ras-transformed fibroblasts (Lin et al. 1996). Gravin is a member of the AKAP (a kinase anchoring protein) family of scaffolding proteins (Nauert et al. 1997; Diviani and Scott 2001; Wong and Scott 2004), which interacts with several proteins required for precise control of morphogenetic cell movements, including the protein kinases PKA (Park et al. 2006) and PKC (Kinoshita et al. 2003; Sheldahl et al. 2003), the protein phosphatase Calcineurin (Saneyoshi et al. 2002), and $\beta 1$,4-Galactosyltransferase (Wassler et al. 2001; Machingo et al. 2006). Gravin has also been implicated in the control of cell shape and behavior through its MARCKS-like domain (myristoylated alanine-rich C kinase substrate), which may link actin to the plasma membrane (Gelman et al. 1998). Expression of Gravin in Src-transformed NIH 3T3 cells results in normal mesenchymal cell morphology and a loss of anchorage-independent growth and Matrigel invasiveness (Lin and Gelman 1997).

Gravin's proposed role as a scaffolding protein that can promote cell shape and behavior changes made it an attractive candidate regulator of embryonic morphogenesis. Since previous studies on the cellular functions of Gravin involved expression in cultured cells, we used zebrafish to determine if Gravin has an essential role in embryogenesis. Using morpholino knockdown experi- ments, we find that loss of Gravin function causes severe axis elongation defects. Intriguingly, in contrast to other mutants and morphants with gastrulation defects, gravin morphant cells migrate normally to the dorsal side, but anterior-posterior extension is severely inhibited. Mesodermal cells lacking Gravin fail to shut down protrusive activity as they reach the dorsal side of the embryo. As a result, cells are unable to undergo the normal medio-lateral intercalative movements required for body axis extension. These results establish Gravin as the first identified regulator of the conversion from migratory to intercalative behavior, and provide important insight into the function of Gravin as a tumor suppressor.

\section{Results}

Gravin is expressed during early zebrafish development

To determine the role of Gravin in early vertebrate development, we cloned the zebrafish ortholog of mammalian gravin. The predicted protein sequence of zebrafish Gravin is similar to the known sequence of human AKAP12 $\beta$ and Xenopus Gravin (Fig. 1A). The N-terminal 1000 amino acids of zebrafish Gravin are 39\% identical to the human sequence, while the $\mathrm{C}$ terminus is poorly conserved, except for a short PKA regulatory subunitbinding domain. The most highly conserved domains of Gravin are three short basic regions, which are implicated in reversible electrostatic interactions with the plasma membrane (Fig. 1B; Streb and Miano 2005).

Gravin is zygotically expressed in zebrafish embryos, with expression first appearing after the mid-blastula transition and peaking at the shield stage (Supplementary Fig. 1), just prior to the onset of convergent extension movements. This peak in gravin expression led us to hypothesize that Gravin plays a critical role in morphogenetic cell movements during gastrulation. During the blastula and gastrula stages, gravin is expressed ubiquitously (Fig. 1C-F). During and after segmentation, gravin expression is restricted to the posterior notochord, adaxial cells, presomitic mesoderm, head mesoderm, nervous tissue, and the vasculature (Fig. 1G-J). The expression pattern we determined for gravin was confirmed by the expression pattern observed for a previously unknown EST (sb:cb27) that corresponds to a fragment of gravin (http://www.zfin.org). Gravin is also expressed in early mouse and frog embryos, although intriguingly, the gastrula-stage expression of Xenopus gravin is restricted to the mesoderm (Gelman et al. 2000; Klingbeil et al. 2001).

\section{Zebrafish Gravin promotes cell shape changes in mammalian cells}

To determine if zebrafish Gravin functions similarly to the mammalian ortholog despite the low overall conservation, we expressed zebrafish GFP-Gravin and human 
A

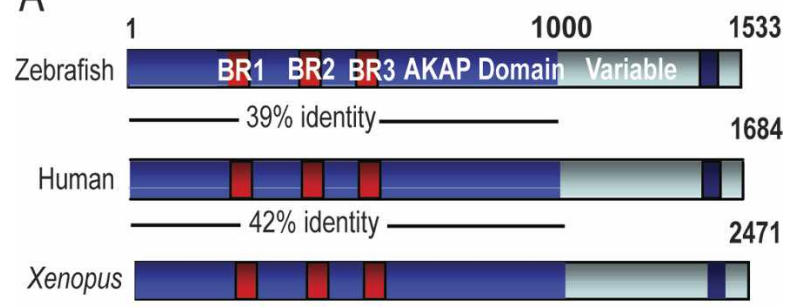

B Basic Repeat 1

$\begin{array}{ll}\text { Fish: } & \text { GFKKIFRFVGFKFTLKKDKNEK } \\ \text { Human: } & \text { GFKKVFKFVGF KTVKKDKTEK }\end{array}$

Basic Repeat 2

Fish: SPFKRFFTQGIFSNLRKKASFKKPK

Human: STFKKFFTQG-WAGWRKKTSFRKPK

Basic Repeat 3

Fish: $\quad$ KSKAHGSPLKKLFTGAGLKKLSSKKHKNKKK

Human: RMKVQGSPLKKLFTSTGLKKLSGKKQKGKR
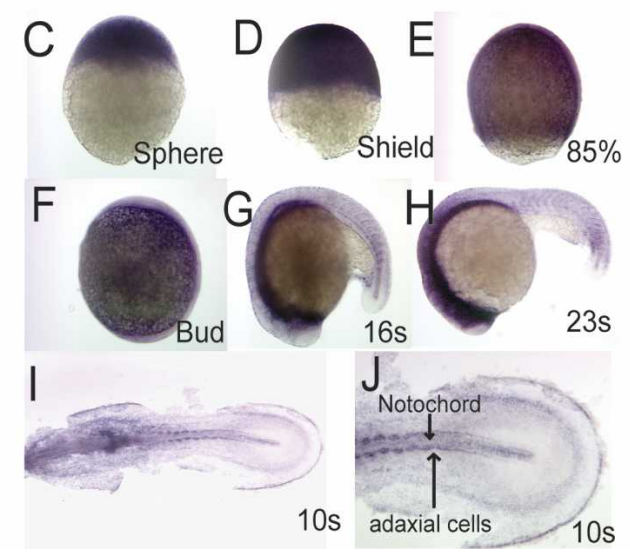

Figure 1. Zebrafish Gravin. A scaffolding protein expressed during early development. (A) Homology of Gravin proteins between zebrafish (Gravin), Xenopus (XGL), and human (AKAP12 $\beta$ ). The percentage of amino acid identity is indicated between the orthologs by domain. (B) Sequence alignment of the basic regions of zebrafish and human Gravin. Charged residues (blue) and phosphorylation sites (red) implicated in membrane interactions are shown. $(C-I)$ Expression of gravin during early development. Gravin is expressed zygotically beginning at the sphere stage $(C)$, and remains ubiquitously expressed during the shield $(D), 85 \%$ epiboly $(E)$, and bud $(F)$ stages. During segmentation, gravin expression becomes restricted to the presomitic mesoderm, head mesoderm, notochord, adaxial cells, vasculature, and nervous system, as seen by lateral view at the 16somite $(G)$ and 23 -somite $(H)$ stages, and by flat mount at the 10 -somite stage $(I, J)$.

GFP-Gravin or a GFP control in COS7 cells. Both human (data not shown) and zebrafish Gravin were predominantly cytoplasmic with perinuclear enrichment (Fig. 2C; data not shown). Also, both human and zebrafish Gravin localized to distinct peripheral membrane puncta as was reported previously for human Gravin (Fig. 2C, white arrows; Streb and Miano 2005). Using a membrane-localized RFP as a control, we determined that these puncta are due to concentrated Gravin and not just artifacts of the fixation (data not shown). The mouse form of Gravin, known as SSeCKS, strongly promotes cell flattening, loss of stress fibers, and increase in lamellapodia-like protrusions when overexpressed (Gelman et al. 1998). COS7 cells expressing either human (data not shown) or zebrafish Gravin (Fig. 2C,D) have a greater cell area and flatter morphology than neighboring untransfected cells or GFP-transfected cells (Fig. 2E). In addition, whereas untransfected or GFP-transfected (Fig. 2F,G) NIH 3T3 cells contain large numbers of stress fibers when plated on poly(D) lysine, expression of zebrafish Gravin (Fig. 2H,I) results in a widespread dissolution of the stress fibers similar to that previously reported for mammalian Gravin (Gelman et al. 1998; quantified in Fig. 2J). These results demonstrate that zebrafish Gravin has the same cell biological properties as the mammalian orthologs.

\section{Gravin is required for proper gastrulation of zebrafish} embryos

To study the role of Gravin during zebrafish development, three antisense morpholino oligonucleotides were designed to gravin (Supplementary Fig. 2B); two were designed to block translation (MO1 and MO2) and one to block splicing (MO3). The efficacy of these morpholinos in blocking translation and splicing of the gravin mRNA was confirmed experimentally (Supplementary Fig. 2A,C).

Injection of each of the gravin morpholinos results in a dose-dependent defect in gastrulation (Supplementary Fig. 2D). Morphant embryos appear grossly similar to control-injected embryos through the bud stage (Fig. $3 \mathrm{G}, \mathrm{H})$. However, during segmentation stages, defects in anterior-posterior extension become apparent (Fig. 3CF). Twelve-somite-stage embryos display reduced anterior-posterior extension, resulting in a greater distance between the head and tail at this stage (Fig. 3E,F, arrows) as well as somite borders that are spaced more closely together (Fig. 3D). At 48 h, morphants display a short, curved body axis with more severe defects in more posterior tissues (Fig. 3A,B). The increasing severity of the gravin MO (morpholino antisense oligonucleotide) phenotype as segmentation occurs has been seen with several convergent extension mutants, including trilobite (Sepich et al. 2000). This occurs because defects in gastrulation impact the ability of somitic and notochordal mesoderm to extend during segmentation, leading to a shortened body axis (Sepich et al. 2000). Strikingly, anterior structures are largely normal in morphant embryos, consistent with the observation that anterior migration of the prechordal plate is unaffected in gravin morphants (Fig. 3G,H). This differs from previously described mutants and morphants that alter convergent extension, which also display defects in prechordal plate migration (Heisenberg et al. 2000; Topczewski et al. 2001; Marlow et al. 2002; Carreira-Barbosa et al. 2003). Mutants and morphants that alter the noncanonical Wnt pathway not only have defects in the convergence and extension of the mesoderm but also produce severe defects in the convergence of endoderm and the organ pre- 
Weiser et al.
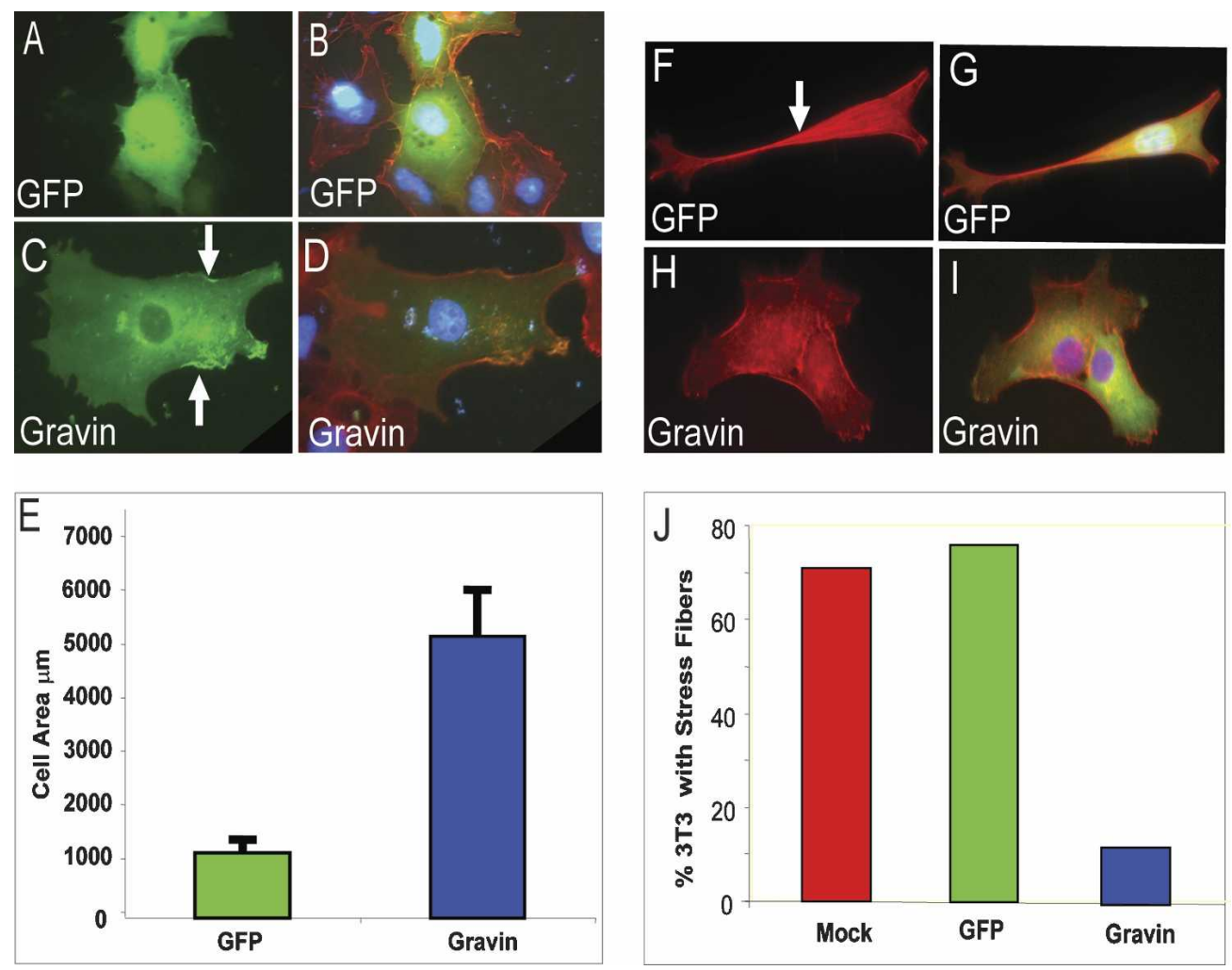

Figure 2. Zebrafish Gravin localizes to the perinucleus and cell periphery in mammalian cells and regulates cell shape. $(A-E)$ COS7 cells were transfected with either GFP alone $(A, B)$ or zebrafish GFP-Gravin $(C, D)$, fixed, and stained with DAPI and Alexa 594phalloidin. GFP alone is shown in the left column, and merged images are in the right column. White arrows indicate sites of peripheral membrane puncta. (E) Cell flattening was assayed by calculating the cell area of GFP- or Gravin-transfected cells using AxioVision 4 software; error bars are standard deviations. $(F-J)$ NIH 3T3 cells transfected with GFP $(F, G)$ or GFP-Gravin $(H, I)$ were fixed and stained with DAPI and phalloidin. $F$ and $H$ show only phalloidin staining, while $G$ and $I$ show phalloidin, GFP, and DAPI. (J) Cells were scored for visible phalloidin-stained stress fibers (see arrow in $F$ ).

cursors, resulting in bifurcated organs (Matsui et al. 2005). While the liver precursors failed to properly converge to the midline in gravin morphants (Fig. 3P,Q), the endoderm, pancreas, and heart progenitors converged to a single domain (Fig. 3J-O). There were, however, morphogenetic defects in the organ primordia in gravin morphants. Heart looping was defective (Fig. 3J,K), whereas pancreas and liver staining was reduced or absent (Fig. $3 \mathrm{~L}, \mathrm{M}, \mathrm{P}, \mathrm{Q})$, potentially due to defects in the induction of pancreas and liver fates. Taken together, these results indicate that Gravin has a role in convergent extension that differs from other factors involved in this process.

To ensure the specificity of the gravin morpholinos, we performed a rescue experiment. Coinjection of zebrafish gravin mRNA (50 pg) at a dose that does not elicit a gastrulation defect together with the splice blocking MO3 (6.5 ng) results in partial rescue of the gastrulation defect, reducing the percentage of severe gastrulation defects from $52 \%$ in morpholino-injected embryos to $22 \%$ (Fig. 3I). This partial rescue is typical of genes involved in morphogenetic movements, since overexpression of these factors also causes convergent extension defects (Topczewski et al. 2001; Jessen et al. 2002; Carreira-Barbosa et al. 2003). This rescue experiment confirms that
Gravin is required for normal morphogenesis in zebrafish.

\section{Extension movements are defective in embryos lacking Gravin}

As described above, the phenotype produced by a loss of gravin suggests that Gravin acts in a different step in gastrulation movements than other factors involved in convergent extension (Heisenberg et al. 2000; Topczewski et al. 2001; Jessen et al. 2002; Kilian et al. 2003). To determine which gastrulation movements are defective in gravin morphants, we used a photo-uncaging strategy to assay the ability of lateral or axial cells to both converge dorsally and extend in the anterior-posterior direction (Sepich and Solnica-Krezel 2005). Small clusters of either lateral or dorsal cells were uncaged at the shield stage (Fig. 4). Four hours later (bud stage), the embryos were then analyzed to determine the extent of cell migration. Cells labeled along the dorsal axis at the shield stage label the notochord, the tailbud, and head mesoderm at the bud stage. Control and gravin morphants produced quite similar results, demonstrating only a minor defect in the convergence and extension of the axial 

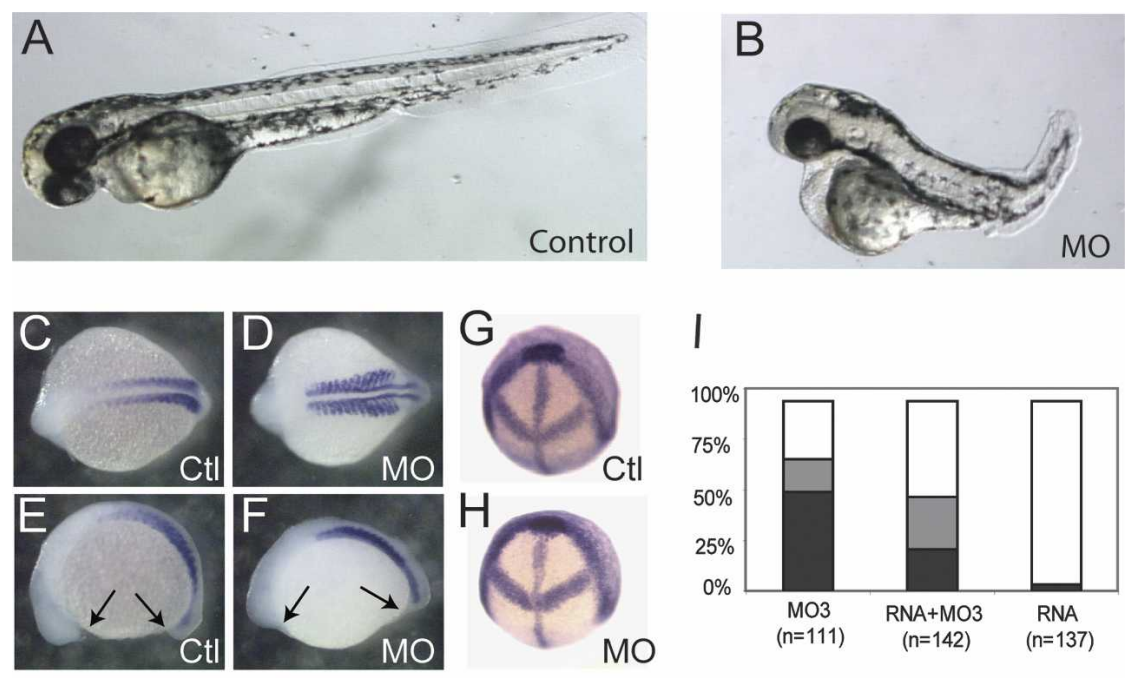

I
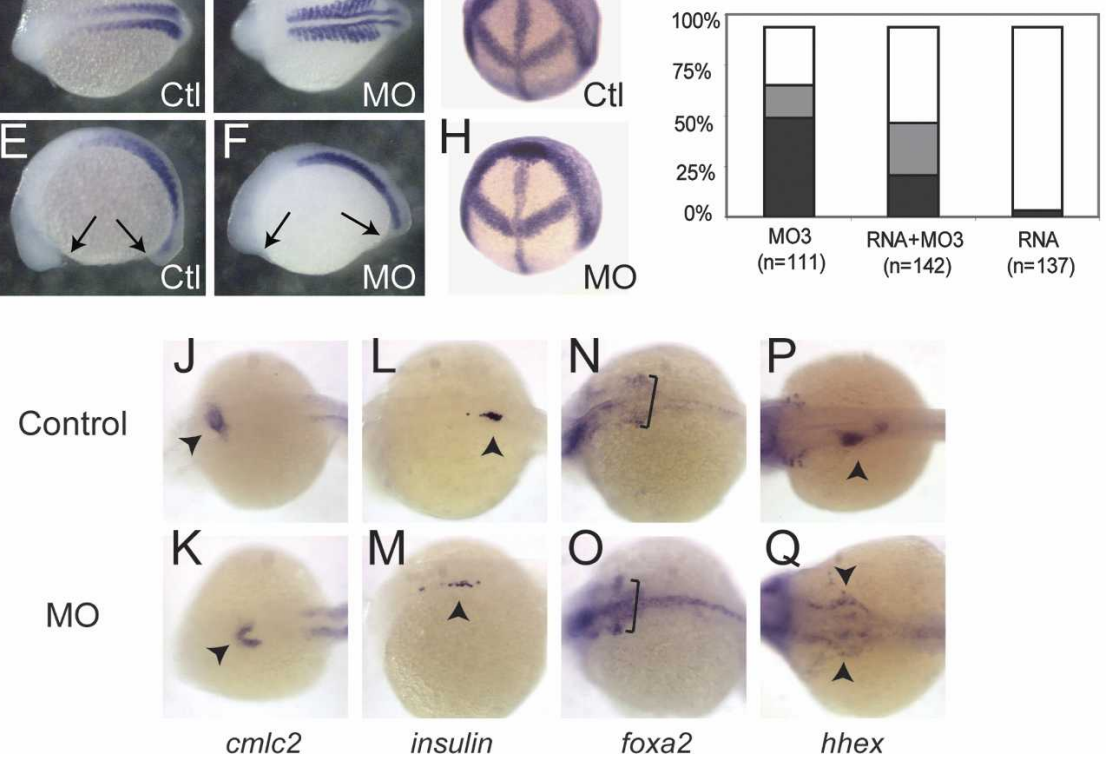

Figure 3. Gravin is required for proper gastrulation of zebrafish embryos. $(A, B)$ The phenotype of gravin-injected $(B)$ and control morpholino-injected $(A)$ embryos at $48 \mathrm{~h}$ post-fertilizaton (hpf). $(C-F)$ The phenotype of gravin morphants was analyzed by in situ hybridization for myoD at the 12-somite stage $(D, F)$ and compared with control-injected embryos $(C, E)$. The arrows mark the extent of the body axis. $(G, H)$ The phenotype of gravin morphants $(H)$ and control embryos $(G)$ at the bud stage stained with hgg1 (to mark the prechordal plate), shh (midline), pax2.1 (midbrain-hindbrain boundary), and $d l_{x} 3$ (neural plate). (I) Coinjection of $50 \mathrm{pg}$ of gravin mRNA partially rescues the gastrulation defect of the splice blocking MO3 (6.5 ng). The $Y$-axis represents the percentage of embryos displaying a severe axis extension defect (black), a moderate phenotype (gray), or normal axis extension (white). $(J-Q)$ Control or morphant embryos were stained with cmlc2 $(J, K)$, insulin $(L, M)$, foxa2 $(N, O)$ and hhex $(P, Q)$ to mark the heart field, pancreas, axial endoderm, and liver, respectively. cells (Fig. 4A-F, quantified in O). In contrast, cells lateral to the notochord, which contribute extensively to the trunk somites, were much more severely affected. Whereas the extent of convergence of these cells was normal, extension was severely perturbed (Fig. 4G-L). Quantification of the movement of lateral cells confirms that dorsal convergence occurs normally, but extension is defective (Fig. 4M,N). Interestingly, while the lateral cells in gravin morphants fully moved toward the dorsal side, once there they were unable to organize into a narrow extended column as was seen with control-injected cells (Fig. 4, cf. L and I). Since paraxial mesoderm showed the strongest defect in morphogenetic movements, we decided to examine the cellular morphology and behavior of the paraxial mesodermal cells.

\section{Gravin promotes cell shape changes in mesodermal cells}

Mesodermal cells undergo a series of cell shape changes during gastrulation. During early gastrulation, they have a nonpolarized morphology and extend multiple protrusions, both short bleb-like and longer lamelliform protrusions (Solnica-Krezel 2006). These cells migrate in an almost random manner but exhibit a slight dorsal bias in net motion (Sepich et al. 2005). By mid-gastrulation, mesodermal cells exhibit a highly polarized morphology and migrate with a very strong dorsal bias, producing a fast net dorsal speed. As mesodermal cells approach the notochord, they change behavior yet again. Instead of migrating autonomously, they begin to act in concert. They become more adherent and protrusive activity is reduced, apparently mediated by contact inhibition (Concha and Adams 1998). These cells then switch from a monopolar mode of migration to exhibit primarily medio-laterally intercalative behavior.

Since gravin morphants are mostly defective in extension and not the dorsal migration of paraxial mesoderm (Fig. 4), we hypothesized that loss of gravin may result in defects in medio-lateral intercalation. To test this, we conducted time-lapse analysis of cell shape in morphant and control embryos throughout gastrulation. Gravin morphant mesodermal cells appeared generally normal during early and mid-gastrulation (data not shown). However, by the bud stage, mesodermal cells adjacent to the notochord displayed severely altered morphology. Control cells were densely packed and exhibited few protrusions (Fig. 5A; Supplementary Movies 1,3). The only protrusions in dorsal paraxial mesodermal cells at this stage were bleb-like protrusions along the notochordal/ paraxial mesodermal boundary and lamelliform protrusions extending into gaps between cells. In contrast, morphant mesodermal cells vigorously extended large numbers of bleb-like protrusions (Fig. 5B; Supplementary Movies 2,4). Some cells extended protrusions in a nonpolarized manner, giving the cells a tumbling morphol- 

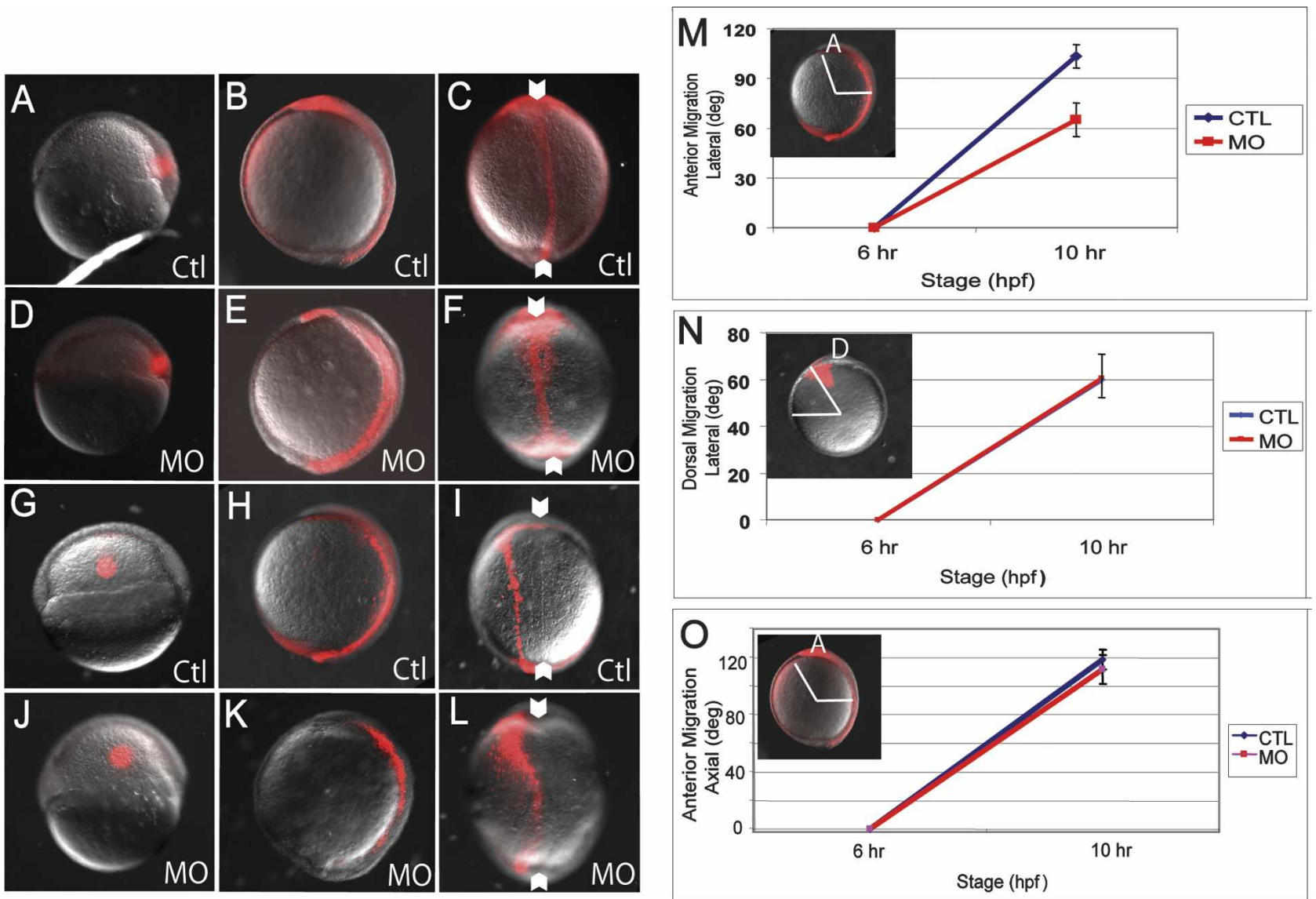

Figure 4. Gravin morphant cells converge dorsally but do not extend. $(A-L)$ Distribution of labeled lateral or dorsal mesodermal and ectodermal cells at the shield and bud stages. The fluorescent dye was uncaged at the shield stage (left panels), and examined $4 \mathrm{~h}$ later at the bud stage in lateral (middle panels) and dorsal (right panels) views. $(A-C)$ Control morpholino-injected (MO3MM, 7.5 ng) embryos, dye uncaged on the dorsal side. $(D-F)$ Gravin morphant embryos (MO3, $7.5 \mathrm{ng})$, dye uncaged on the dorsal side. $(G-I)$ Control morpholino-injected (MO3MM, $7.5 \mathrm{ng}$ ) embryos, dye uncaged on the lateral side (lateral cells were labeled $90^{\circ}$ from dorsal). $(J-L)$ Gravin morphant embryos (MO3, $7.5 \mathrm{ng}$ ), dye uncaged on the lateral side. White arrowheads indicate the notochord. (M,N) Quantification of the dorsal convergence and anterior extension of lateral cells in control and morphant embryos. (M) Anterior extension of lateral mesoderm is defined as the angle between the anterior-most labeled cell and the dorsal side (lateral view, anterior at top). ( $N$ ) Dorsal migration is the angle between the site of activation at the shield stage, and the site of dye-labeled cells at the bud stage (animal pole view, dorsal at top, lateral to the left). (O) Anterior extension of axial mesoderm measured as in $M$. Note that both the convergence of lateral cells and the extension of dorsal cells are unaffected by loss of Gravin; however, extension of lateral cells (including presomitic mesoderm) is severely inhibited. (A) Anterior; (D) dorsal.

ogy similar to the behavior recently described in zebrafish primordial germ cells (Supplementary Movie 2; Blaser et al. 2006). Other cells extended several blebs, which then merged into a leading edge, and those cells then migrated in that direction (Fig. 5B). Whereas control cells averaged less than one bleb per cell over $15 \mathrm{~min}$, gravin morphant cells extended over 12 blebs per cell on average (Fig. 5D). Not only did gravin morphant cells display more protrusive activity than control cells, they also were more motile, with some individual morphant cells, in contrast to control cells, moving more than a cell diameter in $<5 \mathrm{~min}$ (Fig. $5 \mathrm{E}, \mathrm{F}$ ). In addition to excess blebbing, gravin morphant cells were less polarized than control cells. Control paraxial mesodermal cells were intercalating at the bud stage and were lengthened along their medio-lateral axis with a length-to-width ratio of
1.89 (standard deviation 0.34). In contrast, gravin morphant cells were more rounded with a length-to-width ratio of 1.38 (standard deviation 0.29 ). Interestingly, the notochord-presomitic mesodermal boundary formed essentially normally (data not shown), and notochord cells in morphant embryos had similar morphology to notochord cells in control embryos, demonstrating that Gravin plays a greater role in the control of cell behavior in the paraxial mesoderm than in the notochord, despite expression in both tissues.

To determine if the membrane blebbing seen in gravin morphants might somehow be due to apoptosis, we stained morphant and control embryos with acridine orange, a marker of the early stages of cell death. We observed no change in acridine orange staining in gravin morphant embryos compared with control embryos (data 

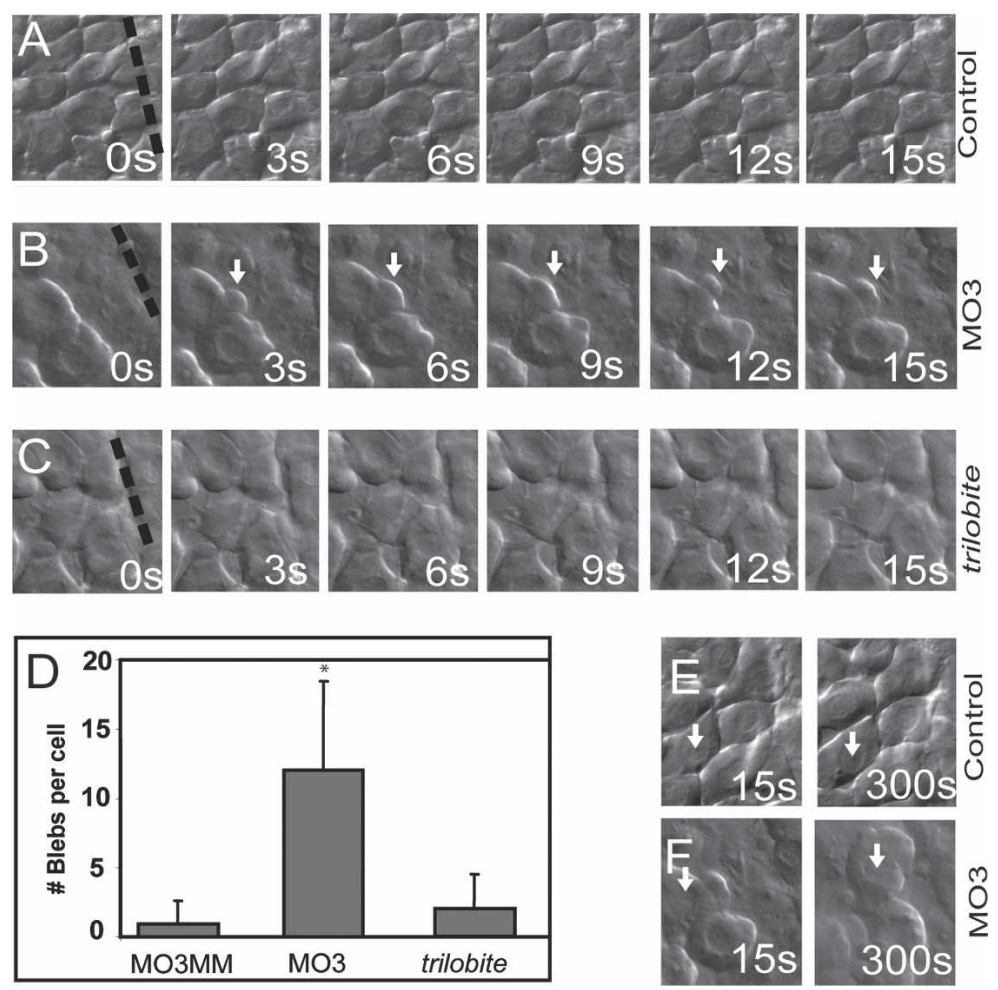

Figure 5. Gravin is required to inhibit protrusions during late gastrulation. Cell shape changes of mesodermal cells between the bud and two-somite stages were compared by time-lapse microscopy. Behavior of dorsal mesodermal cells in control morpholino-injected embryos (MO3MM, $7.5 \mathrm{ng})(A)$, in gravin morpholino-injected embryos (MO3, $7.5 \mathrm{ng}$ ) $(B)$, and in trilobite embryos $(C)$. White arrows indicate bleb-like protrusions, and the dashed black line marks the notochord-somite boundary. (D) Quantification of membrane blebbing behavior of paraxial mesodermal cells in the three conditions shown in $A-C$. The $Y$-axis shows the number of membrane blebs per cell during a 15-min interval. Note the large increase in protrusive activity in gravin morphants relative to control. Longer-term time lapse of cell movements of control (E) and gravin morphant $(F)$ mesodermal cells. The arrow points to the nucleus of the same cell before and after time lapse; (s) seconds; $\left(^{*}\right) p<0.05$. not shown), demonstrating that the ectopic blebbing is caused by changes in cell behavior and not apoptosis.

We next examined the behavior of mesodermal cells in two zebrafish mutants that also exhibit severe convergent extension defects, trilobite and knypek (Topczewski et al. 2001; Jessen et al. 2002), to determine if this altered behavior was a common phenotype of mutants with mesodermal gastrulation defects. Trilobite cells have polarity defects in both the mesoderm and notochord, and have an overall rounder phenotype than wildtype cells (Jessen et al. 2002). However, trilobite cells did not exhibit the ectopic, non-contact-inhibited blebbing of gravin morphants and instead behaved similarly to wild-type cells (Fig. 5C,D). The same results were observed with knypek mutants (data not shown).

Gravin acts through RhoA and Myosin II to elicit cell shape changes

Bleb-like protrusions are a common form of cell migration in cells responding to chemotactic cues (Paluch et al. 2006) and have recently been studied in zebrafish (Blaser et al. 2006) and a variety of other systems (Charras et al. 2005; Langridge and Kay 2006). In zebrafish, for example, the migration of germ cells is entirely mediated by blebbing (Blaser et al. 2006). In contrast, converging mesodermal cells during gastrulation exhibit both lamellipodia and bleb-like protrusions (Concha and Adams 1998). Unlike lamellipodial protrusions, which are driven by actin polymerization, blebs are formed by myosin-based contraction of cortical actin increasing local hydrostatic pressure (Charras et al. 2005). This in- creased pressure provides the force necessary for the cytoplasm to produce a local membrane protrusion without increased actin polymerization. This process depends on the Rho/Rho kinase pathway, which acts to promote the myosin contraction necessary for the blebs to form.

We sought to determine if the ectopic bleb-like protrusions seen in gravin morphants are dependent on the Rho/Rho kinase pathway and actin-myosin contractility as is observed in true locomotive blebbing behavior (Paluch et al. 2006). While mesodermal cells of gravin morpholino-injected embryos display large-scale ectopic blebbing, gravin morphant embryos treated with $50 \mu \mathrm{M}$ myosin inhibitor blebbistatin or $200 \mu \mathrm{M}$ Rho kinase inhibitor III Rockout failed to form most bleb-like protrusions (Fig. 6A,D). These doses were chosen as they both induced convergent extension defects in treated wildtype embryos. However, these treatments did not block all protrusions since filopodia were still present. These results demonstrate that the ectopic blebs produced when Gravin is absent depend on both myosin and Rho kinase function.

Since the ectopic blebbing seen in gravin morphants is dependent on the Rho-myosin pathway, it suggested that one cause of the defect might be due to abnormal regulation of the Rho family GTPases. We therefore asked if there is a synergistic interaction between the Rho family GTPases and a loss of Gravin. We observed a strong synergistic interaction with coinjection of a low dose of gravin morpholino and a low dose of constitutively active rho mRNA. Whereas suboptimal doses of gravin morpholino or mRNA alone caused $<10 \%$ of em- 
Weiser et al.

Figure 6. Ectopic protrusions in Gravin morphants are blocked by inhibition of the Rho/ROCK/Myosin pathway. (A) Embryos injected with a high dose (7.5 ng) of gravin MO3 treated with Rho kinase inhibitor-III just after the shield stage fail to produce blebs. (B) Coinjection of low doses of constitutively active rhoA (1 pg) and gravin MO3 (4 ng) results in excessive membrane blebbing of dorsal mesoderm. White arrows indicate bleb-like protrusions, and the dashed black line marks the notochord-somite boundary. (C) Quantification of the convergent extension (CE) defects caused by low-dose gravin MO3 $(\mathrm{MO} ; 4 \mathrm{ng})$ and constitutively active $r h o A$ (1 pg) or constitutively active rac1 (20 pg). (D) Quantification of the extent of membrane blebbing in embryos injected with a high dose of gravin MO3 (7.5 ng) and treated with Rho kinase inhibitor III (ROCKI) or blebbistatin (bleb) just after the shield stage. $(E)$ Quantification of the extent of membrane blebbing in embryos injected with low doses (4 $\mathrm{ng}$ ) of gravin MO3 and constitutively active $r h o A(1 \mathrm{pg})$.
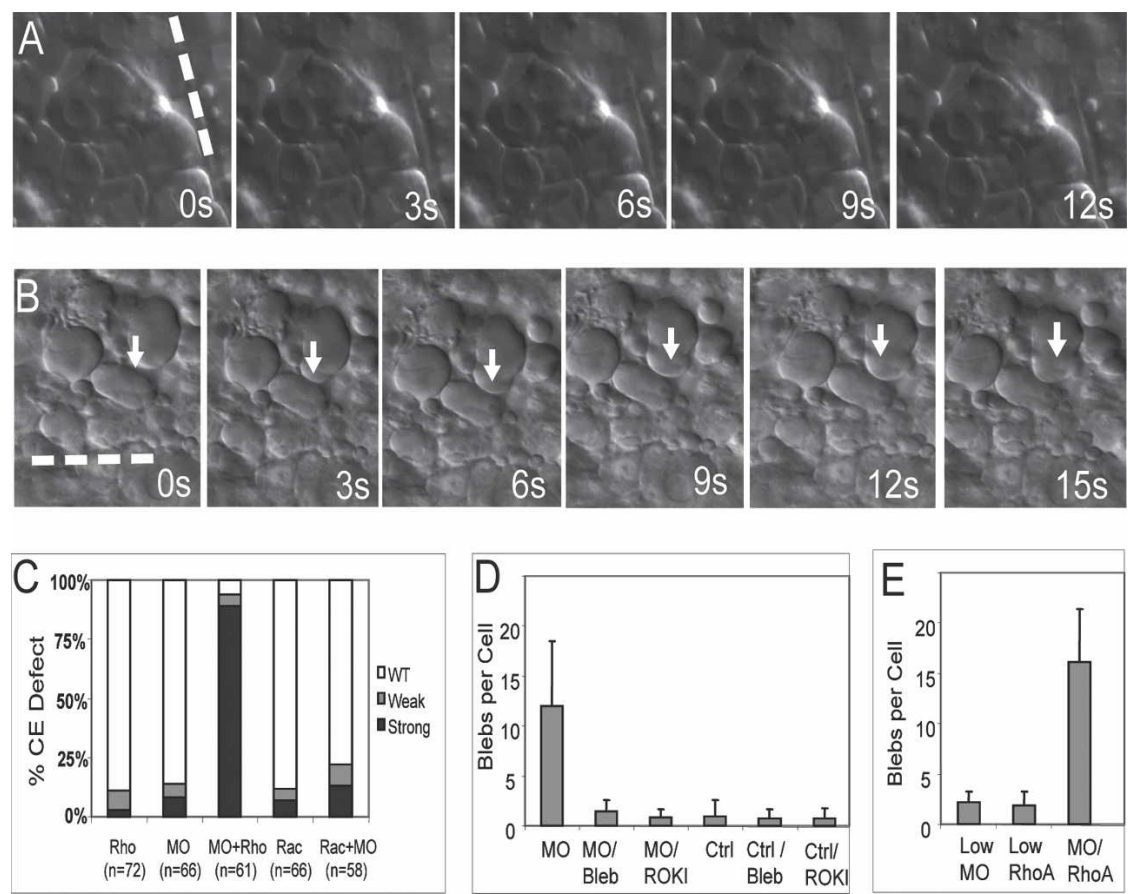

Note that inhibition of ROCK or Myosin II blocks the ectopic blebbing seen in gravin morphants, and that coinjection of low doses of gravin MO3 and constitutively active rhoA mRNA results in synergistic convergent extension defects and membrane blebbing. (s) Seconds.

bryos to have an axis elongation defect, $>90 \%$ of coinjected embryos displayed severe gastrulation defects (Fig. 6C). In contrast, coinjection of constitutively active rac1 (Matsui et al. 2005) and gravin morpholino resulted in only a slight additive effect, indicating no phenotypic interaction (Fig. 6C). Examining the mesodermal cells in embryos coinjected with constitutively active $r$ ho $A$ and gravin morpholino revealed a high level of cellular rounding and blebbing (Fig. 6B,E; Supplementary Movie 5 ), just as was observed with a high level of gravin morpholino, demonstrating synergy at the cellular level between Rho activation and diminished Gravin function. In summary, these results establish an essential role for Gravin in inhibiting Rho-dependent blebbing in order to allow the conversion from convergence to extension.

\section{Discussion}

As embryonic cells undergo morphogenesis, they receive a variety of different inputs and must coordinate their behaviors appropriately. In convergent extension, for example, mesodermal cells first need to move toward the future dorsal midline, where the notochord will eventually form. A large body of studies has shown that noncanonical Wnt signaling plays an essential role in this process (Keller 2002; Veeman et al. 2003; Solnica-Krezel 2006). Once at the midline, however, the cells need to change their behavior dramatically. They need to inhibit the protrusive activity they used while converging and undertake the process of medio-lateral intercalation, which extends the body axis (Concha and Adams 1998;
Jessen et al. 2002; Marlow et al. 2002; Glickman et al. 2003; Solnica-Krezel 2006). How cells know when to change from convergence to extension is unknown, but it is likely that direct cell-cell contact is involved, since the cells become tightly packed as they accumulate on the dorsal side of the embryo. Here we identify Gravin as a key modulator of this process.

\section{Gravin is required for mesodermal cell behavior during zebrafish gastrulation}

We found that depletion of Gravin using three different morpholinos results in a severe dose-dependent defect in body extension. The body axis extension defect in these embryos is as severe as the defect found in the zebrafish gastrulation mutants trilobite and knypek (Topczewski et al. 2001; Jessen et al. 2002). There are, however, several differences between the classic convergent extension phenotype of trilobite and knypek and the gravin morphant phenotype. We found that the anterior end of the embryo formed normally in gravin morphants, unlike in other convergent extension mutants. Also the convergence of anterior structures, such as the organ primordia was normal in gravin morphants, unlike in mutants and morphants that alter the noncanonical Wnt pathway. The severe defects in overall body axis elongation and the defect in somite morphogenesis caused us to focus on the cell behavior of presomitic mesodermal cells during gastrulation. We found that the dorsal directed migration of lateral paraxial mesodermal cells in gravin morphants was similar to control-injected em- 
bryos, and that the extension of the notochordal mesoderm was also only mildly affected by gravin depletion. Both of these processes have been widely implicated in convergent extension movements, and both are severely affected in many morphants and mutants with gastrulation defects (Heisenberg et al. 2000; Topczewski et al. 2001; Jessen et al. 2002; Marlow et al. 2002; Yamashita et al. 2002). However, in gravin morphants, it is the extension behavior of presomitic mesodermal cells that is severely affected, resulting in the shortened body axis.

Our studies lend support to the emerging view that convergence and extension are mechanistically distinct processes. For example, no tail mutant embryos display severe loss of convergence of notochordal cells during the late gastrulation and early segmentation stages, whereas notochord extension proceeds normally (Glickman et al. 2003). Has 2 morphant embryos display a severe loss of lateral mesodermal cell migration and overall convergence, yet extension of dorsal tissues is unaffected (Bakkers et al. 2004). Morpholino knockdown of flamingo, the zebrafish homolog of a Drosophila PCP gene, affects both convergence and extension (Formstone and Mason 2005). However, in chimeric analysis, flamingo morphant cells converged normally but failed to extend when transplanted into wild-type hosts, indicating a cell-autonomous function that primarily affects extension, in addition to a non-cell-autonomous role that affects both convergence and extension. Thus, while gravin morphants have similarities to other morphants and mutants that alter convergent extension, the gravin phenotype is unique in primarily or exclusively affecting extension.
We examined the cell behaviors of individual presomitic mesodermal cells during gastrulation to determine the cause of the extension defect. Normally, cells exhibit high levels of protrusive activity as they converge toward the dorsal side, but then shut down this activity as they transition to the extension phase (Trinkaus et al. 1992; Concha and Adams 1998; Jessen et al. 2002). In the absence of Gravin, mesodermal cells on the dorsal side continued to exhibit extremely high levels of protrusive activity. Interestingly, this protrusive activity was primarily rapidly forming and short-lived bleb-like protrusions, which are also observed in mesodermal cells undergoing convergence behavior (Blaser et al. 2006). Using cell transplantation, we determined that gravin morphant cells adjacent to the notochord in wildtype hosts bleb profusely, whereas wild-type cells transplanted into gravin morphant hosts do not show increased protrusive activity (D.C. Weiser and D. Kimelman, unpubl.). Also, gravin morphant cells transplanted into other tissues such as ectoderm do not show excess protrusive activity. This indicates that the effect of gravin loss is cell-autonomous, and the excess protrusive activity is specific to paraxial mesoderm.

We propose that the major role of Gravin in early embryogenesis is to shut down the highly protrusive activity that is required by converging cells, which allows these cells to undergo extension (Fig. 7A). In the absence of Gravin, the continued high level of bleb-like protrusive activity impedes extension, producing embryos with severely altered phenotypes (Fig. 7B). It is intriguing that mesodermal cells in gravin morphants mostly shut down the lamellipodial protrusions seen during fast dor-
A

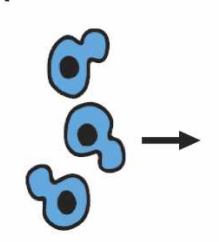

Slow Dorsal Migration

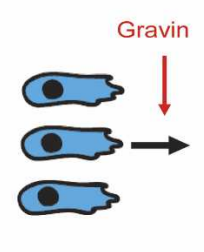

Fast Dorsal Migration

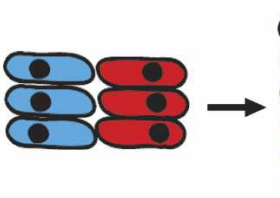

Packing Inhibition of Protrusions Intercalation
B

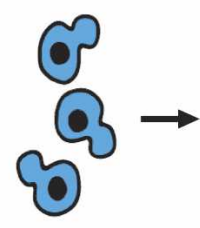

Slow Dorsal Migration

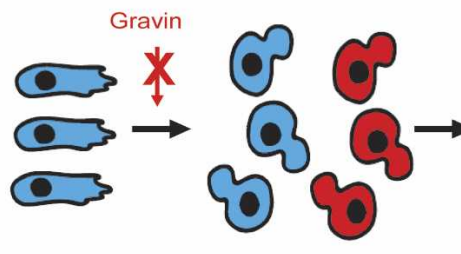

Fast Dorsal Migration
No Packing Excessive Protrusions

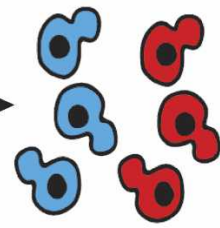

Failure of Intercalation

C

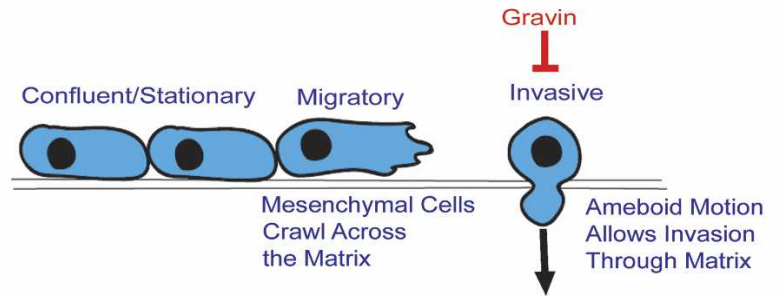

Figure 7. A model of Gravin's role in regulating cell behaviors during gastrulation and metastasis. $(A)$ Mesodermal cells undergo a series of cell behavior changes during gastrulation, progressing from nonpolar highly migratory cells, to polarized dorsal migratory cells, to intercalating cells extending few protrusions. $(B)$ In the absence of Gravin, mesodermal cells fail to suppress their protrusive activity, leading to large-scale ectopic blebbing. These highly protrusive cells are then unable to undergo complex morphogenetic movements, and body axis extension is inhibited. (C) A model of Gravin function in tumor metastasis, where Gravin acts as a tumor suppressor by inhibiting the amoeboid-type movements in tumor cells that contribute to invasive cell behaviors by enhancing migration through the ECM. 
sal migration after they had converged toward the dorsal side of the embryo. This raises the possibility that another factor, working in concert with Gravin, inhibits lamellipodia prior to extension.

This study is the first to report a mutant or morphant that fails to inhibit protrusive activity, which we propose is a unique property of gravin morphants. Not only are gravin morphants among the rare class of mutants and morphants that primarily or exclusively affect extension, but we did not observe a defect in the inhibition of protrusive activity in trilobite and knypek mutants, which also produce severe convergent extension defects. An understanding of Gravin's intracellular functions will provide important clues as to how the transition from convergence to extension occurs. As a scaffolding protein that binds many regulatory factors and is membrane-associated, Gravin is well positioned to communicate signals from the extracellular environment to processes occurring inside the mesodermal cells. We suggest that as the mesodermal cells converge on the dorsal side, they come into close contact with each other, which sends a signal mediated by Gravin to inhibit protrusive activity. Our data suggest that precise regulation of Rho family GTPases and downstream effectors may be an essential aspect of Gravin's role. Rho family GTPases are required for morphogenetic cell movements and either gain or loss of function of these GTPases or downstream effectors causes severe morphogenetic defects in both convergence and extension (Marlow et al. 2002; Habas et al. 2003; Matsui et al. 2005). We found that inhibition of Rho kinase or Myosin II, which is regulated by Rho kinase, blocked all ectopic blebbing in gravin morphants, suggesting that a key role for Gravin is to transiently shut down Rho kinase activity and myosin-based contraction in order to make the transition to the extension phase. In agreement with this, activation of Rho synergistically enhanced the convergent extension defects and blebbing phenotype produced by a reduction in Gravin. Intriguingly, a very recent report showed that Gravin can block activation of Rho family GTPases by Src and prevent Rho-dependent formation of invasive protrusions, such as podosomes, in a cultured cell system (Gelman and Gao 2006), supporting our idea that Gravin is working through Rho regulation. Because of its many advantages as an experimental system, zebrafish embryos should be very useful for understanding how Gravin regulates intracellular processes governing the transition from convergence to extension.

\section{Implications for tumorogenesis and metastasis}

A key event in metastasis of epithelial cells is the conversion of cells to a migratory state, which is termed the epithelial-mesenchymal transition. This typically involves a down-regulation of cadherin-based adhesion, and the acquisition of integrin-based migration involving polarized protrusions and focal adhesions (Friedl and Wolf 2003). These focal-adhesive regions recruit surface proteases such as matrix metalloproteinases (MMPs), which locally degrade the extracellular matrix (ECM), enhancing the ability of cells to move through the ECM. Recently, however, it has become clear that tumor cells can also move using amoeboid, or bleb-like, movements (Friedl 2004; Wolf and Friedl 2006). While some tumor cell lines naturally move through a three-dimensional matrix using amoeboid movements, even cells that naturally prefer to use classical mesenchymal movements can be converted to amoeboid movement when the surface proteases are inhibited or when ROCK is activated (Sahai and Marshall 2003; Wolf et al. 2003). This mesenchymal-amoeboid transition has been proposed to be an escape mechanism for tumor cells that allows them to continue migrating even when the surface proteases are inhibited, which may explain in part why MMP inhibitors have failed to live up to their promise as valuable cancer therapeutics (Wolf and Friedl 2006).

Gravin is an in vivo tumor suppressor down-regulated by several oncogenes in vitro and in multiple human tumors in vivo (Xia et al. 2001). The gravin gene also maps to a chromosomal hotspot for deletion in highly metastatic human prostate cancers (Xia et al. 2001). Ectopic expression of Gravin in v-Src-transformed fibroblasts inhibits several behavioral changes including reduced adhesiveness, increased cell rounding, invasive protrusive activity, matrigel invasiveness, loss of anchorage-dependent growth, and loss of contact inhibition of growth, all hallmarks of a metastatic phenotype (Xia et al. 2001; Charras et al. 2006; Gelman and Gao 2006). Interestingly, while expression of Gravin blocks invasive and metastatic behaviors, it does not affect two-dimensional cell migration in scratch assays (Xia et al. 2001), which parallels our studies that a loss of Gravin does not affect convergence in zebrafish embryos.

Our studies in zebrafish embryos showing that a loss of Gravin causes cells to abnormally produce ROCK-dependent bleb-like movements after they have converged to the midline suggests that a key function of Gravin in cells is to inhibit amoeboid-type movements (Fig. 7C). In this view, a reduction or loss of Gravin in tumor cells would enhance their ability to use amoeboid-based migration. While amoeboid-based migration can allow cells to escape pharmacological inhibition (Wolf and Friedl 2006), we suggest that tumor cells that can use both mesenchymal-type migration and amoeboid-based migration may have an advantage in metastasis since they can migrate through a wider range of extracellular environments than cells that use only mesenchymal-based migration. For example, in vivo imaging of the MTLn3 metastatic breast cancer cells revealed that these cells become elongated and polarized as they move toward blood vessels, but then switch to amoeboid movements as they crawl into the blood vessels (Lai et al. 2005). In this sense, highly metastatic cells may be very similar to the early zebrafish mesodermal cells, which can use both types of migratory behavior during gastrulation.

Sahai and Marshall (2003) have proposed that RhoROCK signaling promotes metastasis by promoting amoeboid-type migration. A loss of Gravin would favor this conversion since we found that it promotes ROCKdependent bleb-like movements. Intriguingly, recent 
work demonstrates that ectopic Gravin expression in fibroblasts blocks the formation of Rho-dependent protrusions (Gelman and Gao 2006), supporting the contention that Gravin works in opposition to Rho/ROCK signaling. We therefore suggest that a loss or reduction of Gravin promotes a highly metastatic state, by enhancing the potential for amoeboid-type migration.

In conclusion, we have demonstrated that Gravin plays a critical role in controlling complex cell behavior changes in mesodermal cells undergoing gastrulation. Consistent with its defined role in cancer metastasis, Gravin is not a simple promoter or inhibitor of directed cell migration but is required for proper control of complex morphogenetic cell movements. In the absence of Gravin, cells display phenotypes of excessive Rho kinase and Myosin-dependent membrane blebbing. These cells are therefore unable to undergo the medio-lateral intercalative behaviors required for axis elongation. This study provides novel insight into the molecular and cellular mechanisms required for morphogenetic cell movements during vertebrate development and shows that zebrafish is an excellent model system for examining how cells use both mesenchymal- and amoeboid-type movements.

\section{Materials and methods}

\section{Zebrafish}

Wild-type WIK/AB zebrafish (Danio rerio) embryos were obtained through natural spawning and were maintained, injected, and staged according to established procedures (Westerfield 1993). Trilobite and knypek embryos were the kind gift of the laboratory of Randall Moon.

\section{Cloning of zebrafish gravin, plasmids, and morpholinos}

Detailed information on gravin cloning and plasmid construction are available upon request. The GenBank accession number for zebrafish gravin is EF539208. Messenger RNA was synthesized using the mMessage Machine Kit (Ambion) following the manufacturer's instructions. Morpholinos and mRNA were injected into zebrafish embryos at the one-cell stage. The following gravin morpholinos were used in the study: MO1 (5'-CTGT TAGAGTTATTGTCCCAAGCAT-3'), MO2 (5'-AGATTTGCC GTTATCTTTCGCTTT-3'), MO3 (5'-TCTTACCTGTTAGAG TTATTGTCCC-3'), Mismatch-MO2 (5'-AGATTTCCCCTTA ATGTTTCCCTTT-3'), and Mismatch-MO3 (5' ${ }^{\prime}$-TGTTACGTG TTACAGTTTTTGTGCC-3').

\section{Mammalian tissue culture}

COS7 and NIH 3T3 cells (obtained from the American Type Culture Collection) were maintained in DMEM with $10 \%$ fetal bovine serum and DMEM with $10 \%$ bovine calf serum, respectively, and cultured in $5 \% \mathrm{CO}_{2}$. Cells were transfected with the indicated cDNA using Fugene (Roche). Cells were then cultured overnight and fixed in $4 \%$ paraformaldehyde, washed, and stained with Alexa 594 phalloidin (Molecular Probes) to label actin filaments and 4',6-diamidino-2-phenylindole (DAPI; Molecular Probes) to label the nucleus.

\section{In situ hybridization}

Whole-mount in situ hybridization was performed using digoxigenin-labeled antisense RNA probes and visualized using anti- digoxigenin Fab fragments conjugated to alkaline phosphatase (Roche Molecular Biochemicals). DNA templates were linearized and transcribed with either SP6 or T7 RNA polymerases. Embryos were staged, fixed, processed, and hybridized as described (Westerfield 1993).

\section{Uncaging experiment}

One-cell-stage embryos were injected with $0.5 \%$ caged carboxyrhodamine dextran (10,000 MW; Molecular Probes). At the shield stage, the dye was uncaged using a Zeiss Axiovert 200M microscope at $40 \times$, a 10 -sec UV photoactivation, and a closed pinhole. Embryos were then imaged at the bud stage for analysis of convergence and/or extension as described (Sepich and Solnica-Krezel 2005).

\section{Time-lapse microscopy}

Embryos were mounted in a drop of $1.5 \%$ methyl cellulose in embryo medium in a glass-bottomed dish. Time-lapse recordings were then performed with a Zeiss Axiovert 200M microscope using AxioVision 4 software, DIC optics, and a $40 \times$ objective at $28^{\circ} \mathrm{C}$.

\section{Quantification of membrane blebbing}

Twenty to 40 paraxial mesodermal cells per embryo, in the superficial-most layer of mesoderm within six cell diameters of the notochord, were scored individually during a 15-min time lapse. The number of bleb-like protrusions per cell was counted, and the data were reported as either the percentage of cells blebbing in $15 \mathrm{~min}$ or the number of blebs per cell per $15 \mathrm{~min}$. Error bars are 1 standard deviation.

\section{Quantification of cell shape changes in vitro}

The cell area of GFP or Gravin-transfected COS7 cells was calculated using the cell area tool in AxioVision 4 software. Stress fiber quantification was performed on fixed 3T3 cells stained with phalloidin. The cells were scored as stress fiber positive if two or more stress fibers were clearly visible and scored as negative if one or fewer were visible.

\section{Modulation of Rho-myosin signaling}

One-cell-stage embryos were injected with gravin morpholino and grown to the shield stage, dechorionated, and treated with $50 \mu \mathrm{M}$ myosin inhibitor blebbistatin and $200 \mu \mathrm{M}$ Rho kinase inhibitor III Rockout or a DMSO control. At the bud stage, the embryos were assayed for membrane blebbing of paraxial mesodermal cells. To increase Rho GTPase activity, 1 pg of Xenopus RhoA V14 mRNA or 20 pg of human Rac1 V14 mRNA were injected into one-cell-stage embryos.

\section{Acknowledgments}

We gratefully acknowledge Dr. Steve Hauschka for the very kind use of his microscope and tissue culture facility, and thank John Scott for human cDNA, Randall Moon for supplying mutant embryos, Kathryn Eisenmann and Arthur Alberts for a valuable discussion, Hong Shin and Didier Stainer for supplying plasmids, and Susan Walsh, Benjamin Martin, and Ashley Webb for critical reading of the manuscript. This work was supported by NIH grant RO1HD27262 to D.K. and an NRSA fellowship F32HD053189-01 to D.C.W. 


\section{References}

Bakkers, J., Kramer, C., Pothof, J., Quaedvlieg, N.E., Spaink, H.P., and Hammerschmidt, M. 2004. Has2 is required upstream of Rac1 to govern dorsal migration of lateral cells during zebrafish gastrulation. Development 131: 525-537.

Blaser, H., Reichman-Fried, M., Castanon, I., Dumstrei, K., Marlow, F.L., Kawakami, K., Solnica-Krezel, L., Heisenberg, C.P., and Raz, E. 2006. Migration of zebrafish primordial germ cells: A role for Myosin contraction and cytoplasmic flow. Dev. Cell 11: 613-627.

Carreira-Barbosa, F., Concha, M.L., Takeuchi, M., Ueno, N., Wilson, S.W., and Tada, M. 2003. Prickle 1 regulates cell movements during gastrulation and neuronal migration in zebrafish. Development 130: 4037-4046.

Charras, G.T., Yarrow, J.C., Horton, M.A., Mahadevan, L., and Mitchison, T.J. 2005. Non-equilibration of hydrostatic pressure in blebbing cells. Nature 435: 365-369.

Charras, G.T., Hu, C.K., Coughlin, M., and Mitchison, T.J. 2006. Reassembly of contractile actin cortex in cell blebs. $J$. Cell Biol. 175: 477-490.

Choi, S.C. and Han, J.K. 2002. Xenopus Cdc42 regulates convergent extension movements during gastrulation through Wnt/Ca ${ }^{2+}$ signaling pathway. Dev. Biol. 244: 342-357.

Concha, M.L. and Adams, R.J. 1998. Oriented cell divisions and cellular morphogenesis in the zebrafish gastrula and neurula: A time-lapse analysis. Development 125: 983-994.

Daggett, D.F., Boyd, C.A., Gautier, P., Bryson-Richardson, R.J., Thisse, C., Thisse, B., Amacher, S.L., and Currie, P.D. 2004. Developmentally restricted actin-regulatory molecules control morphogenetic cell movements in the zebrafish gastrula. Curr. Biol. 14: 1632-1638.

Diviani, D. and Scott, J.D. 2001. AKAP signaling complexes at the cytoskeleton. J. Cell Sci. 114: 1431-1437.

Formstone, C.J. and Mason, I. 2005. Combinatorial activity of Flamingo proteins directs convergence and extension within the early zebrafish embryo via the planar cell polarity pathway. Dev. Biol. 282: 320-335.

Friedl, P. 2004. Prespecification and plasticity: Shifting mechanisms of cell migration. Curr. Opin. Cell Biol. 16: 14-23.

Friedl, P. and Wolf, K. 2003. Tumour-cell invasion and migration: Diversity and escape mechanisms. Nat. Rev. Cancer 3: 362-374.

Gelman, I.H. and Gao, L. 2006. SSeCKS/Gravin/AKAP12 metastasis suppressor inhibits podosome formation via RhoAand Cdc42-dependent pathways. Mol. Cancer Res. 4: 151158.

Gelman, I.H., Lee, K., Tombler, E., Gordon, R., and Lin, X. 1998. Control of cytoskeletal architecture by the src-suppressed C kinase substrate, SSeCKS. Cell Motil. Cytoskeleton 41: $1-17$.

Gelman, I.H., Tombler, E., and Vargas Jr., J. 2000. A role for SSeCKS, a major protein kinase C substrate with tumour suppressor activity, in cytoskeletal architecture, formation of migratory processes, and cell migration during embryogenesis. Histochem. J. 32: 13-26.

Glickman, N.S., Kimmel, C.B., Jones, M.A., and Adams, R.J. 2003. Shaping the zebrafish notochord. Development 130: 873-887.

Habas, R., Kato, Y., and He, X. 2001. Wnt/Frizzled activation of Rho regulates vertebrate gastrulation and requires a novel Formin homology protein Daam1. Cell 107: 843-854.

Habas, R., Dawid, I.B., and He, X. 2003. Coactivation of Rac and Rho by Wnt/Frizzled signaling is required for vertebrate gastrulation. Genes \& Dev. 17: 295-309.

Hall, A. 1998. Rho GTPases and the actin cytoskeleton. Science
279: 509-514.

Heisenberg, C.P. and Tada, M. 2002. Zebrafish gastrulation movements: Bridging cell and developmental biology. Semin. Cell Dev. Biol. 13: 471-479.

Heisenberg, C.P., Tada, M., Rauch, G.J., Saude, L., Concha, M.L., Geisler, R., Stemple, D.L., Smith, J.C., and Wilson, S.W. 2000. Silberblick/Wnt11 mediates convergent extension movements during zebrafish gastrulation. Nature 405: 76-81.

Jessen, J.R., Topczewski, J., Bingham, S., Sepich, D.S., Marlow, F., Chandrasekhar, A., and Solnica-Krezel, L. 2002. Zebrafish trilobite identifies new roles for Strabismus in gastrulation and neuronal movements. Nat. Cell Biol. 4: 610-615.

Jopling, C. and den Hertog, J. 2005. Fyn/Yes and non-canonical Wnt signalling converge on RhoA in vertebrate gastrulation cell movements. EMBO Rep. 6: 426-431.

Keller, R. 2002. Shaping the vertebrate body plan by polarized embryonic cell movements. Science 298: 1950-1954.

Kilian, B., Mansukoski, H., Barbosa, F.C., Ulrich, F., Tada, M., and Heisenberg, C.P. 2003. The role of Ppt/Wnt5 in regulating cell shape and movement during zebrafish gastrulation. Mech. Dev. 120: 467-476.

Kinoshita, N., Iioka, H., Miyakoshi, A., and Ueno, N. 2003. PKC $\delta$ is essential for Dishevelled function in a noncanonical Wnt pathway that regulates Xenopus convergent extension movements. Genes \& Dev. 17: 1663-1676.

Klingbeil, P., Frazzetto, G., and Bouwmeester, T. 2001. Xgravinlike $(\mathrm{Xgl})$, a novel putative a-kinase anchoring protein (AKAP) expressed during embryonic development in Xenopus. Mech. Dev. 100: 323-326.

Lai, S.L., Chang, C.N., Wang, P.J., and Lee, S.J. 2005. Rho mediates cytokinesis and epiboly via ROCK in zebrafish. Mol. Reprod. Dev. 71: 186-196.

Langridge, P.D. and Kay, R.R. 2006. Blebbing of Dictyostelium cells in response to chemoattractant. Exp. Cell Res. 312: 2009-2017.

Lin, X. and Gelman, I.H. 1997. Reexpression of the major protein kinase C substrate, SSeCKS, suppresses v-src-induced morphological transformation and tumorigenesis. Cancer Res. 57: 2304-2312.

Lin, X., Tombler, E., Nelson, P.J., Ross, M., and Gelman, I.H. 1996. A novel src- and ras-suppressed protein kinase C substrate associated with cytoskeletal architecture. I. Biol. Chem. 271: 28430-28438.

Lin, F., Sepich, D.S., Chen, S., Topczewski, J., Yin, C., SolnicaKrezel, L., and Hamm, H. 2005. Essential roles of Go12/13 signaling in distinct cell behaviors driving zebrafish convergence and extension gastrulation movements. J. Cell Biol. 169: $777-787$.

Machingo, Q.J., Fritz, A., and Shur, B.D. 2006. A $\beta 1,4-$ galactosyltransferase is required for convergent extension movements in zebrafish. Dev. Biol. 297: 471-482.

Marlow, F., Topczewski, J., Sepich, D., and Solnica-Krezel, L. 2002. Zebrafish Rho kinase 2 acts downstream of Wnt11 to mediate cell polarity and effective convergence and extension movements. Curr. Biol. 12: 876-884.

Matsui, T., Raya, A., Kawakami, Y., Callol-Massot, C., Capdevila, J., Rodriguez-Esteban, C., and Izpisua Belmonte, J.C. 2005. Noncanonical Wnt signaling regulates midline convergence of organ primordia during zebrafish development. Genes \& Dev. 19: 164-175.

Miyakoshi, A., Ueno, N., and Kinoshita, N. 2004. Rho guanine nucleotide exchange factor xNET1 implicated in gastrulation movements during Xenopus development. Differentiation 72: 48-55.

Mlodzik, M. 2002. Planar cell polarization: Do the same mecha- 
nisms regulate Drosophila tissue polarity and vertebrate gastrulation? Trends Genet. 18: 564-571.

Nauert, J.B., Klauck, T.M., Langeberg, L.K., and Scott, J.D. 1997. Gravin, an autoantigen recognized by serum from myasthenia gravis patients, is a kinase scaffold protein. Curr. Biol. 7: 52-62.

Paluch, E., Sykes, C., Prost, J., and Bornens, M. 2006. Dynamic modes of the cortical actomyosin gel during cell locomotion and division. Trends Cell Biol. 16: 5-10.

Park, E., Kim, G.H., Choi, S.C., and Han, J.K. 2006. Role of PKA as a negative regulator of PCP signaling pathway during Xenopus gastrulation movements. Dev. Biol. 292: 344-357.

Sahai, E. and Marshall, C.J. 2003. Differing modes of tumour cell invasion have distinct requirements for Rho/ROCK signalling and extracellular proteolysis. Nat. Cell Biol. 5: 711719.

Saneyoshi, T., Kume, S., Amasaki, Y., and Mikoshiba, K. 2002. The Wnt/calcium pathway activates NF-AT and promotes ventral cell fate in Xenopus embryos. Nature 417: 295-299.

Sepich, D.S. and Solnica-Krezel, L. 2005. Analysis of cell movements in zebrafish embryos: Morphometrics and measuring movement of labeled cell populations in vivo. Methods Mol. Biol. 294: 211-233.

Sepich, D.S., Myers, D.C., Short, R., Topczewski, J., Marlow, F., and Solnica-Krezel, L. 2000. Role of the zebrafish trilobite locus in gastrulation movements of convergence and extension. Genesis 27: 159-173.

Sepich, D.S., Calmelet, C., Kiskowski, M., and Solnica-Krezel, L. 2005. Initiation of convergence and extension movements of lateral mesoderm during zebrafish gastrulation. Dev. Dyn. 234: 279-292.

Sheldahl, L.C., Slusarski, D.C., Pandur, P., Miller, J.R., Kuhl, M., and Moon, R.T. 2003. Dishevelled activates $\mathrm{Ca}^{2+}$ flux, PKC, and CamKII in vertebrate embryos. J. Cell Biol. 161: 769-777.

Solnica-Krezel, L. 2006. Gastrulation in zebrafish-All just about adhesion? Curr. Opin. Genet. Dev. 16: 433-441.

Streb, J.W. and Miano, J.M. 2005. Cross-species sequence analysis reveals multiple charged residue-rich domains that regulate nuclear/cytoplasmic partitioning and membrane localization of AKAP 12 (SSeCKS/Gravin). J. Biol. Chem. 280: 28007-28014.

Topczewski, J., Sepich, D.S., Myers, D.C., Walker, C., Amores, A., Lele, Z., Hammerschmidt, M., Postlethwait, J., and Solnica-Krezel, L. 2001. The zebrafish glypican knypek controls cell polarity during gastrulation movements of convergent extension. Dev. Cell 1: 251-264.

Trinkaus, J.P., Trinkaus, M., and Fink, R.D. 1992. On the convergent cell movements of gastrulation in Fundulus. J. Exp. Zool. 261: 40-61.

Veeman, M.T., Axelrod, J.D., and Moon, R.T. 2003. A second canon. Functions and mechanisms of $\beta$-catenin-independent Wnt signaling. Dev. Cell 5: 367-377.

Wallingford, J.B., Rowning, B.A., Vogeli, K.M., Rothbacher, U., Fraser, S.E., and Harland, R.M. 2000. Dishevelled controls cell polarity during Xenopus gastrulation. Nature 405: 8185.

Wassler, M.J., Foote, C.I., Gelman, I.H., and Shur, B.D. 2001. Functional interaction between the SSeCKS scaffolding protein and the cytoplasmic domain of $\beta 1,4$-galactosyltransferase. J. Cell Sci. 114: 2291-2300.

Westerfield, M. 1993. The zebrafish book: A guide for the laboratory use of zebrafish (Brachydanio rerio). University of Oregon Press, Eugene.

Wolf, K. and Friedl, P. 2006. Molecular mechanisms of cancer cell invasion and plasticity. Br. J. Dermatol. 154 (Suppl. 1):
$11-15$.

Wolf, K., Mazo, I., Leung, H., Engelke, K., von Andrian, U.H., Deryugina, E.I., Strongin, A.Y., Brocker, E.B., and Friedl, P. 2003. Compensation mechanism in tumor cell migration: Mesenchymal-amoeboid transition after blocking of pericellular proteolysis. J. Cell Biol. 160: 267-277.

Wong, W. and Scott, J.D. 2004. AKAP signalling complexes: Focal points in space and time. Nat. Rev. Mol. Cell Biol. 5: 959-970.

Xia, W., Unger, P., Miller, L., Nelson, J., and Gelman, I.H. 2001. The Src-suppressed C kinase substrate, SSeCKS, is a potential metastasis inhibitor in prostate cancer. Cancer Res. 61: 5644-5651.

Yamashita, S., Miyagi, C., Carmany-Rampey, A., Shimizu, T., Fujii, R., Schier, A.F., and Hirano, T. 2002. Stat 3 controls cell movements during zebrafish gastrulation. Dev. Cell 2: 363 375. 


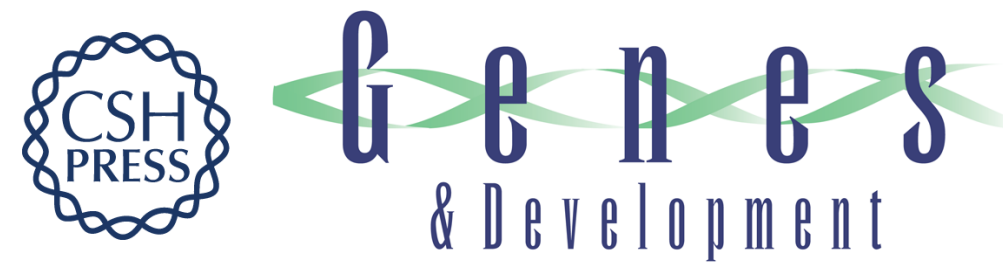

\section{Gravin regulates mesodermal cell behavior changes required for axis elongation during zebrafish gastrulation}

Douglas C. Weiser, Ujwal J. Pyati and David Kimelman

Genes Dev. 2007, 21:

Access the most recent version at doi:10.1101/gad.1535007

Supplemental
Material http://genesdev.cshlp.org/content/suppl/2007/06/12/21.12.1559.DC1

References This article cites 58 articles, 19 of which can be accessed free at:

http://genesdev.cshlp.org/content/21/12/1559.full.html\#ref-list-1

License

Email Alerting

Receive free email alerts when new articles cite this article - sign up in the box at the top

Service

right corner of the article or click here.

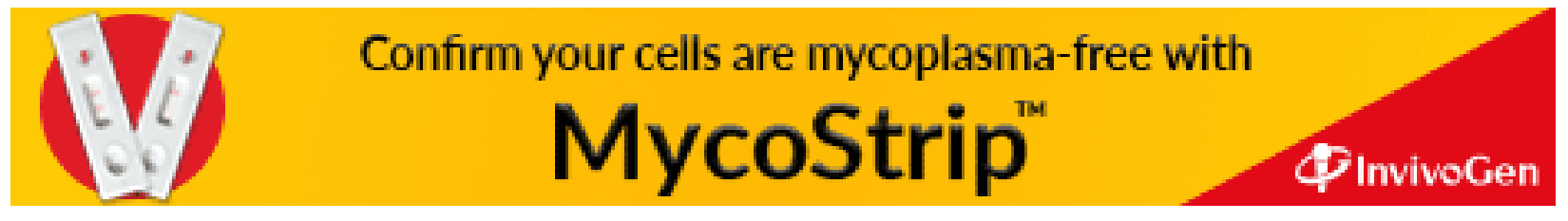

